\title{
Gait speed among older participants enrolled in an evidence-based fall risk reduction program: a subgroup analysis
}

\section{Jinmyoung Cho ${ }^{1,2} *$, Matthew Lee Smith ${ }^{3,2}$, Tiffany E. Shubert ${ }^{4}$, Luohua Jiang ${ }^{5,6}$, SangNam Ahn ${ }^{7,2}$ and Marcia G. Ory ${ }^{2}$}

${ }^{1}$ Center for Applied Health Research, Baylor Scott \& White Health, Temple, TX, USA

2 Department of Health Promotion and Community Health Science, Texas A\&M Health Science Center School of Public Health, College Station, TX, USA

${ }^{3}$ Department of Health Promotion and Behavior, The University of Georgia, Athens, GA, USA

${ }^{4}$ School of Medicine, University of North Carolina, Chapel Hill, NC, USA

${ }^{5}$ Department of Epidemiology, School of Medicine, University of California, Irvine, CA, USA

${ }^{6}$ Department of Epidemiology and Biostatistics, Texas A\&M Health Science Center School of Public Health, College Station, TX, USA

7 Division of Health Systems Management and Policy, The University of Memphis, Memphis, TN, USA

\section{Edited by:}

Sanjay P. Zodpey, Public Health

Foundation of India, India

\section{Reviewed by:}

Cheryll Diann Lesneski, The University of North Carolina at Chapel Hill, USA

Preeti Negandhi, Indian Institute of

Public Health Delhi, India

\section{*Correspondence:}

Jinmyoung Cho, Department of Health Promotion and Community Health Science, Texas A\&M Health Science Center, School of Public Health, Center for Applied Health Research, Baylor Scott and White Health, MS-01-501, 2401 S 31st

Street, Temple, TX 76508, USA e-mail: jcho@sw.org, jinmyoung.cho@sph.tamhsc.edu
Background: Functional decline is a primary risk factor for institutionalization and mortality among older adults. Although community-based fall risk reduction programs have been widely disseminated, little is known about their impact on gait speed, a key indicator of functional performance. Changes in functional performance between baseline and postintervention were examined by means of timed up and go (TUG), a standardized functional assessment test administered to participants enrolled in A Matter of Balance/Volunteer Lay Leader (AMOB/NLL) model, an evidence-based fall risk reduction program.

Methods: This study included 71 participants enrolled in an AMOBNLL program in the Brazos Valley and South Plain regions of Texas. Paired $t$-tests were employed to assess program effects on gait speed at baseline and post-intervention for all participants and by subgroups of age, sex, living status, delivery sites, and self-rated health. The Bonferroni correction was applied to adjust inflated Type I error rate associated with performing multiple $t$-tests, for which $p$-values $<0.0042$ (i.e., $0.5 / 12$ comparisons) were deemed statistically significant.

Results: Overall, gait speed of enrolled participants improved from baseline to postintervention ( $t=3.22, p=0.002$ ). Significant changes in TUG scores were observed among participants who lived with others $(t=4.45, p<0.001)$, rated their health as excellent, very good, or good ( $t=3.05, p=0.003)$, and attended program workshops at senior centers $(t=3.52, p=0.003)$.

Conclusion: Findings suggest community-based fall risk reduction programs can improve gait speed for older adults. More translational research is needed to understand factors related to the effectiveness of fall risk reduction programs in various populations and settings.

\section{Keywords: older adults, A Matter of Balance/Volunteer Lay Leader model, timed up and go}

\section{FALLS AMONG OLDER ADULTS}

Falls among older adults are a serious public health problem in America (1). Approximately one-fourth of older adults aged 80 years and older experience at least two falls per year (2-4). As the risk of falling increases with advanced age, dramatic escalations in fall-related morbidity, hospitalization, institutionalization, and mortality can be expected to accompany the aging of the population (5). Direct annual medical care costs related to falls have been estimated at almost $\$ 20$ billion and are projected to increase sharply in the coming decades $(6,7)$.

Various demographic, functional, and health factors are known to increase the risk for falling among older adults (8). These factors include age $(2,4)$, being female $(9,10)$, a prior history of falls $(2$, $4)$, gait and mobility deficits $(2,4,9,11)$, and poor self-reported health status $(9,10)$. In addition to personal characteristics, particular attention has been paid to the environmental circumstances surrounding falls, such neighborhood environments or program delivery settings (12).

Fall-prevention programs and integration of prevention services have been shown to decrease fall recurrence (13) and reduce health-care costs (14). However, literature about the effectiveness of evidence-based fall-prevention programs for improving objectively measured functional performance has been limited for programs delivered in the community by lay facilitators. 
Given its ease of use and standardization, gait speed, often called "timed up and go (TUG)," has been frequently used to assess functional performance as an outcome measurement for effective interventions $(15,16)$. Many studies have demonstrated a strong relationship between gait speed and fall-related risk, health and functional status, institutionalization, and mortality among older adults (17-19). To address the existing research gaps, the overall goal of this study was to examine improvement in functional performance among older participants enrolled in A Matter of Balance/Volunteer Lay Leader (AMOB/VLL) model, an evidence-based fall risk reduction program.

\section{A MATTER OF BALANCE/VOLUNTEER LAY LEADER FALL RISK REDUCTION PROGRAM}

A Matter of Balance (AMOB), established at the Roybal Center for Enhancement of Late-Life Function at Boston University, is an evidence-based program to reduce risk of falls among older adults (20). The effectiveness of the AMOB program was originally tested through a randomized clinical trial, which showed positive improvements in physical activity and mobility control (21). Derived from the original program, the AMOB/VLL model has been adapted for widespread community dissemination in various health and aging sectors $(22,23)$. Delivered by trained lay-led facilitators in 38 states, it is presented in 2-h sessions for 8 weeks. One hour is taught by a physical therapist. This hour focuses on the role of exercise in fall prevention. It is not meant to be a standalone session, but rather an introduction for older adults to build upon. At the end of AMOB, participants are more likely to exercise and intended activity (21). Each session includes specific goals for older adults to reduce the risk of falling and continue remaining active and independent (24). The major goals of the program are as follows: to make participants perceive control, to increase their confidence, and to learn falls are controllable. The design of intervention targets behavior change and emphasizes building fall self-efficacy and setting goals for increasing physical activity through lectures, group discussions, various problem-solving and role-playing activities, exercise and assertiveness training, and individual assignments (24).

Since 2006, a well-established infrastructure has facilitated the delivery of the intervention to older adults in Texas (24). The network for aging services arranged a signed agreement with the Texas Association of Area Agencies on Aging (AAA) for implementation of the AMOB/VLL program in many AAA regions. The program targets low-income older minority adults and their caregivers and focuses on reaching a diverse population in a large geographic area. Residential facilities, health-care institutions, public health departments, faith-based organizations, business sectors, and local government were included as partners with the Texas AAA sites to build fall-prevention capacity $(24,25)$.

\section{PURPOSE OF STUDY}

Although community-based health promotion programs result in for improvement in falls efficacy, overall health status, and increased physical activities $(11,26)$, less is known about their impact on physical performance (i.e., TUG) among older participants. The purposes of this study were to (a) assess the changes in physical performance measured by the TUG test from baseline to post-intervention and (b) compare the improvement in physical performance by personal characteristics and delivery sites. A conceptual model for this study is shown in Figure 1. This model depicts the fall risk reduction program as an intervention that can have positive effects on changes in physical performance. If participation in AMOB results in improved efficacy as well as improved physical performance, physical therapists may want to include AMOB as a program for appropriate patients. To better understand who may benefit

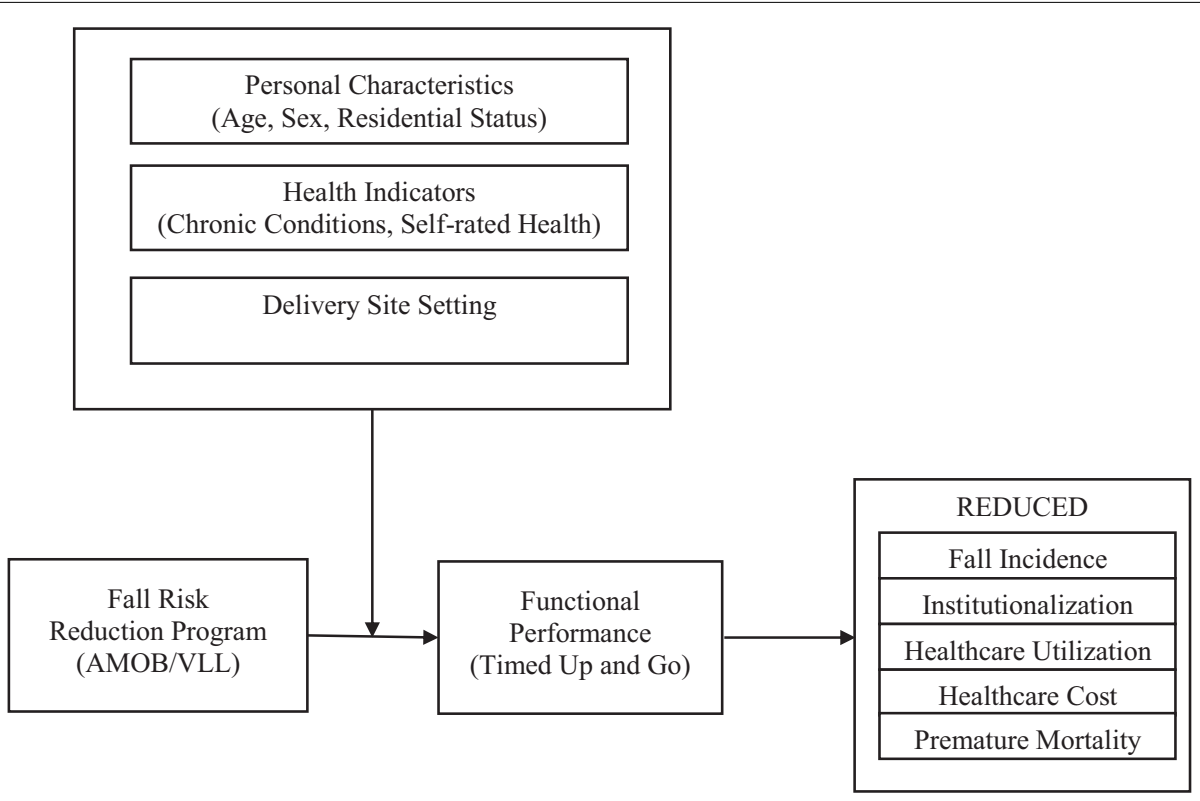

FIGURE 1 | Conceptual model. 
the most from this program, personal characteristics and delivery sites act as moderators between the fall risk reduction program (as illustrated by the AMOB/VLL model) and physical performance as measured by the TUG test. In turn, the TUG measurements are associated with long-term improvements in reduced health-care use and costs as well as enhanced health and well-being.

\section{METHODS}

\section{PROCEDURES AND PARTICIPANTS}

A total of 301 participants enrolled in the Texas AMOB/VLL fall risk reduction program between September 2007 and April 2009 in two regional AAAs: Brazos Valley and South Plain. Although functional assessment was optional in the statewide AAA delivery of the AMOB/VLL program, assessments were conducted in some classes that taught these two regions. Workshop leaders were trained in assessment procedures and performed assessments in eight of the AMOB/VLL classes, which served as the basis for this study. A total of 171 participants who attended classes in these two regions but who were not assessed using the TUG were excluded; thus, 76 participants completed the TUG test at baseline and postintervention, whereas 54 participants did not complete the test at both times. Boxplots were used to screen for outliers for TUG scores from both baseline and post-intervention. Results indicated the presence of three outliers, who were then omitted. An additional two cases reporting an "other" ethnic group were excluded to maximize racial and ethnic homogeneity of participants for this study. As a result, only non-Hispanic White participants were included in this study. Figure 2 shows the recruitment flow diagram, indicating that 71 participants were included in study analyses.

\section{MEASURES}

\section{Timed up and go test}

The TUG test, introduced in 1991 by Podsiadlo and Richardson (27), has been used extensively for over a decade to predict fall risk and to examine functional mobility among older adults $(26,28)$. It assesses the time in seconds that participants required to "rise from a standard arm chair, walk at your typical or normal pace to a line on the floor 3 meters away, turn, return, and sit down again" (p. 64) (27). This test was validated to test physical functioning and mobility among community-dwelling older adults (26) and showed high predictive validity with the Berg Balance Scale (27). Those who completed the TUG tasks in more than $14 \mathrm{~s}$ also showed lower scores on the Berg Balance Scale, which was associated with higher risk for institutionalization (26).

Enrolled in two Texas

counties delivering

AMOB/VLL $(n=301)$

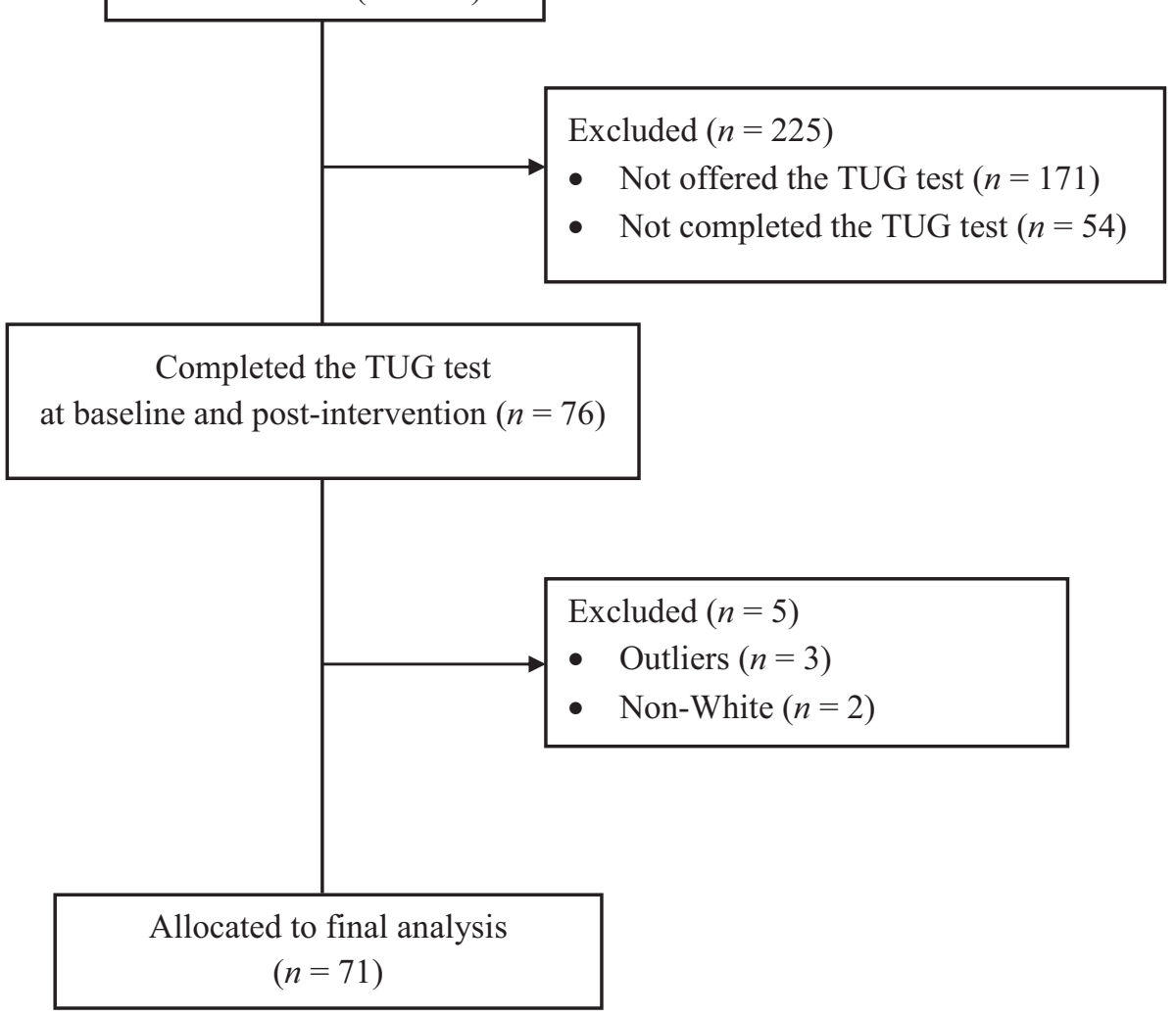

FIGURE 2 | Diagram for study participants inclusion. 


\section{Personal characteristics}

Age was coded as a continuous variable based on a participant's birth date and ranged from 56 to 95 . The age was then categorized into three groups for the purpose of the study: young-old (up to 69 years), mid-old (from 70 to 79 years), and old-old ( 80 years and older). Sex was scored 0 if the participant was male and 1 if the participant was female. Living status was scored 1 if participants lived with others and 0 if they lived alone. Self-rated health was included. Self-rated health, a single item measuring in which participants rate current status of their overall health, has been widely used as a significant predictor of physical and psychological health such as mortality or functionality among various populations (29-32). Many studies have shown that the single item is a reliable and valid measure reflecting objective health status (e.g., cardio-cerebral vascular diseases, visual impairment) (3234). At baseline, participants were also asked to self-report their perceived health status: "Would you say that in general your health is poor, fair, good, very good, or excellent?" For comparisons of self-rated health, the responses were divided into two categories (i.e., poor/fair vs. good/very good/excellent).

\section{Delivery sites}

To compare outcomes at the various settings in which the AMOB/VLL program was conducted in the Brazos Valley and South Plain regions, delivery site types were obtained from administrative data. Delivery site categories included senior centers, community centers, faith-based organizations, residential facilities, and other Parks Department facilities. For comparisons of delivery sites, five sites were categorized into three groups: senior centers and community centers, residential facilities, and others.

\section{Data analysis}

The paired $t$-test was employed to compare mean TUG scores for all participants pre- and post-intervention. Statistical significance was examined at the level of 0.05 for this test. Then, a series of paired $t$-tests were employed to compare the TUG scores by subgroups: age groups, sex, residential status, delivery sites, and self-rated health. Bonferroni's correction was applied for subgroup (12 groups) comparisons to adjust the inflated Type I error rate associated with performing multiple $t$-tests, for which $p$-values $<0.0042$ (i.e., $0.5 / 12$ comparisons) were deemed statistically significant. Statistical analyses were conducted with SPSS statistical software (version 20.0). As an indicator of practical significance, Cohen's $d$ standardized effect sizes were calculated to compare intervention effects from baseline to post-intervention within each group.

\section{RESULTS}

\section{SAMPLE CHARACTERISTICS}

Table 1 summarizes characteristics of study participants. The average age of the study participants was $77.8(\mathrm{SD}=9.3)$ years old. The majority of participants was female $(80.6 \%)$, and more than half the participants lived with others $(56.1 \%)$. Over three quarters of the participants rated their health good, very good, or excellent $(75.4 \%)$. Most participants had at least one chronic health problem $(84.4 \%)$. Within the two regional AAAs, the AMOB/VLL program was implemented in residential facilities (52.1\%), senior
Table 1 | Characteristic of the study participants

\begin{tabular}{lc}
\hline Characteristics & Frequency (\%) \\
\hline Age, mean (SD) (range: 56-95) & $77.8(9.3)$ \\
Sex & \\
Male & $13(19.4)$ \\
Female & $54(80.6)$ \\
Residential status & \\
Living alone & $29(43.9)$ \\
Living with others & $37(56.1)$ \\
Self-rated health & \\
Excellent & $5(7.7)$ \\
Very good & $17(26.2)$ \\
Good & $27(41.5)$ \\
Fair & $15(23.1)$ \\
Poor & $1(1.5)$ \\
Numbers of chronic condition & \\
None & $11(15.5)$ \\
1-2 & $44(61.9)$ \\
3+ & $16(22.5)$ \\
Delivery sites & \\
Senior centers & $15(21.1)$ \\
Community centers & $2(2.8)$ \\
Residential facilities & $37(52.1)$ \\
Faith-based organizations & $9(12.7)$ \\
Other-parks department facilities & $8(11.3)$ \\
&
\end{tabular}

Different numbers of missing cases were observed for each variable. Missing cases were excluded from calculations and analyses.

centers $(21.1 \%)$, faith-based organizations (12.7\%), other Parks Department facilities (11.3\%), and community centers $(2.8 \%)$.

\section{CHANGES IN TIMED UP AND GO TEST}

Before the paired $t$-test for the TUG score was conducted, the TUG scores at baseline and post-intervention were observed. Almost a third of participants $(28.2 \%)$ at baseline and $22.5 \%$ of participants at post-intervention performed slower than $14 \mathrm{~s}$, which represents a critical value on the TUG test. Table 2 presents results of the paired $t$-tests for TUG scores among all AMOB/VLL program participants and by subgroups from baseline to postintervention. Among all participants, the average TUG score at baseline was $12.89(\mathrm{SD}=5.08)$ and changed to $11.95(\mathrm{SD}=4.30)$ at post-intervention $(t=3.22, p=0.002)$. When comparing TUG score changes by subgroup, three significant improvements were found. First, participants who lived with others showed significant changes in TUG scores from baseline $(M=12.61, \mathrm{SD}=5.92)$ to post-intervention $(M=11.32, \mathrm{SD}=5.04), t=4.45, p<0.001$. The effect size (Cohen's $d$ ) was 0.23 . Second, participants who attended the AMOB/VLL program at senior centers or community centers showed statistically significant improvement in TUG scores from $14.96(\mathrm{SD}=7.20)$ at baseline to $13.30(\mathrm{SD}=6.21)$ at post-intervention, $t=3.52, p=0.003$. Cohen's $d$ was 0.25 . Third, those who perceived their health good, very good, or excellent showed significant improvement in TUG scores: 12.77 ( $\mathrm{SD}=5.41)$ 
Table 2 | Average TUG scores in pre- and post-test by groups

\begin{tabular}{|c|c|c|c|c|c|}
\hline & Pre (SD) & Post (SD) & $t$-Value & $p$ & Cohen's $d$ \\
\hline Total participants & $12.89(5.08)$ & $11.95(4.30)$ & 3.22 & 0.002 & 0.25 \\
\hline \multicolumn{6}{|l|}{ Age groups } \\
\hline Young-old $(n=14)$ & $9.74(2.21)$ & $8.89(2.06)$ & 2.60 & 0.018 & 0.40 \\
\hline Mid-old $(n=16)$ & $11.67(3.43)$ & $10.83(2.80)$ & 2.25 & 0.040 & 0.27 \\
\hline Old-old ( $n=34)$ & $14.85(6.06)$ & $13.82(5.00)$ & 2.20 & 0.035 & 0.19 \\
\hline Male $(n=13)$ & $12.41(4.00)$ & $11.53(3.59)$ & 1.33 & 0.208 & 0.23 \\
\hline Female $(n=54)$ & $12.95(5.45)$ & $12.08(4.62)$ & 2.77 & 0.008 & 0.17 \\
\hline \multicolumn{6}{|l|}{ Living status } \\
\hline Living alone $(n=29)$ & $12.93(4.05)$ & $12.66(3.44)$ & 0.52 & 0.605 & 0.07 \\
\hline Living with others $(n=37)$ & $12.61(5.92)$ & $11.32(5.04)$ & 4.45 & $<0.001$ & 0.23 \\
\hline \multicolumn{6}{|l|}{ Delivery sites } \\
\hline \multicolumn{6}{|l|}{ Self-rated health groups } \\
\hline Excellent/VG/good ( $n=57)$ & $12.77(5.41)$ & $11.87(4.56)$ & 3.05 & 0.003 & 0.18 \\
\hline Fair/poor $(n=11)$ & $13.08(2.78)$ & $11.86(3.36)$ & 1.25 & 0.240 & 0.40 \\
\hline
\end{tabular}

Different numbers of missing cases were observed for each variable. Missing cases were excluded from calculations and analyses.

at baseline and $11.87(\mathrm{SD}=4.56)$ at post-intervention, $t=3.05$, $p=0.003$. The effect size was 0.18 .

\section{DISCUSSION}

The primary objective in this study was to examine changes in functional performance between baseline and post-intervention among participants enrolled in the Texas AMOB/VLL fall risk reduction program. Several important findings emerged from this study. First, the average score for all participants' walking speed assessed with the standardized TUG test improved from baseline to post-intervention. These findings demonstrate that this fall risk reduction program can improve gait speed among old participants in addition to its previously reported benefits for falls efficacy and fear of falling (22). Second, subgroup comparisons showed significant improvements among those who rated their health more positively, lived with others, and attended program workshops in senior centers or community centers. These findings reveal that improvement in functional performance (i.e., TUG) may be directly associated with participating in a fall risk reduction program for these subgroups.

The most significant aspect of this study was the incorporation of an objectively measured functional assessment to compare participant improvement based on self-reported measures. Because most measures from evidence-based programs have been based on self-reported information, such as healthrelated quality of life, number of falls, and number of chronic conditions within the previous week or month, a couple of other researchers noted that self-reported measures might produce recall bias as a data collection limitation $(23,35,36)$. Using a standardized functional assessment test (i.e., TUG) can contribute to the validation of previous findings that reported improvements in the ability to perform important social and role functions $(23,36)$.

Findings of the current study also highlight the importance of physical health, social, and environmental correlates to enhance the effectiveness of the evidence-based program. First, the analyses revealed that better perception of health was associated with significant improvement on the TUG test. It is obvious that those who perceived their health to be of better status showed significant improvement because these individuals may be more likely to have fewer chronic conditions and may be less influenced by daily activity limitations. However, the largest standardized Cohen's effect size (Cohen's $d=0.40$ ) was notably observed among participants who self-reported their health to be fair/poor despite the lack of statistical significance of the TUG score change. In other words, those with worse health status may show larger changes in functional assessments because they have greater opportunity for improvement, whereas those healthier participants who score high at baseline have little room for improvement (36). This finding points to the need for future research to increase understanding of the functional improvements of individuals of different health status levels and detect underlying statistical effects, such as regression to the mean.

Second, the significant improvement in gait speed based on residential status emphasizes the importance of social correlates on the effectiveness of the evidence-based program. Results showed significant improvement in functional performance from baseline to post-intervention among participants who live with others. This finding is consistent with previous studies that has shown the significant relationship between physical activities and support from family or friends $(37,38)$. Living with others is likely to prevent older adults from social isolation, which has been identified as a 
barrier to physical activity (38). This finding may also indicate that participants who lived with others had social support mechanisms that may have encouraged them to attend more AMOB/VLL program sessions (i.e., received more intervention dose) and engage in recommended physical activities outside class time.

Third, findings of this study suggest delivery settings in which evidence-based programs that are implemented can enhance physical performance among old participants. Participants who attended workshops in senior centers or community centers showed significant improvement in TUG scores from baseline to post-intervention, which may highlight an environmental benefit for delivering evidence-based programs to older adults in these group settings. This finding may be associated with the notion that these participants were healthier upon program enrollment or that the location of the delivery site was more accessible, which increased their attendance (i.e., intervention does) and led to significant improvement.

In an attempt to disseminate widely fall-prevention programs, the Texas AAA sites have continued to build fall-prevention capacity by partnering with the public health network and others to establish programs in various settings, such as residential facilities, faith-based organizations, workplace setting, health-care institutions, public health departments, and governmental facilities (25). Although other studies have identified differences in program outcomes by delivery site types (35), further investigation is warranted to understand the influence of delivery site on functional assessment measures among lay-led fall-prevention programs.

\section{LIMITATIONS AND IMPLICATIONS}

The findings of this study showed significant TUG score changes associated with this fall risk reduction program; however, a few notable limitations were associated with this study. First, this study included only 71 study participants. The small sample size may limit generalization of our findings to other populations. Second, as stated in the procedures and participant section, older minority adults were excluded from this study because too few participated for meaningful analyses. Although Texas is a geographically large and demographically diverse state, the two minority group cases were intentionally excluded to yield a homogeneous sample of participants. If enough minorities had been available for meaningful comparisons, we may find ethnic difference in functional performance among more diverse groups of participants. Finally, if objective method of rating current health status (e.g., biomarkers) was used in the fall risk reduction program instead of self-rated health, the result may provide an association between health status and functional capacity among old participants.

The findings from the current study have considerable implications for future research on translational studies. Although this study provides an important view of the use of TUG tests in a community-based fall risk reduction program, additional research is needed to link functional assessment scores to the actual fall experience, subsequent health-care use, and the availability of supportive environments illustrated in our conceptual model of fall risk behaviors, interventions, and long-term outcomes. First, the capacity for objective functional measurement among community-dwelling older adults should be built into evidencebased fall-prevention programs. For example, instructor manuals for lay leaders should include a training session about objective functional measurement. Such provider training is important for maintaining measurement necessary for research assessment. In recognition of the importance of objective measurements for purposes of both research and programing, this type of training has been built into CDC's State Fall Prevention Program (39). Furthermore, future studies should focus on participants' degree of disability to examine more comprehensively the effectiveness of evidence-based fall risk reduction programs in different populations. Considering the extent of the disability or investigating the difference in physical performance between fallers and non-fallers may suggest detailed strategies to promote physical activity for older adults with various baseline functional levels. Also, more translational research is needed to understand potential modifiable and non-modifiable correlates related to effectiveness of fall risk reduction programs on functional performance within various populations and settings.

\section{ACKNOWLEDGMENTS}

A Matter of Balance/Voluntary Lay Leader (AMOB/VLL) is a major program activity in the Aging Texas Well's Texas Healthy Lifestyles Initiative. Statewide implementation is supported by the Department of Aging and Disability Services and administered through the Texas Association of Area Agencies on Aging. The evaluation is conducted by the Texas A\&M Health Science Center School of Public Health. We recognize faculty support from The Center for Community Health Development, which is a member of the Prevention Research Centers Program, supported by the Centers for Disease Control and Prevention cooperative agreement number 5U48 DP000045. The findings and conclusions in this article are those of the author(s) and do not necessarily represent the official position of Department of Aging and Disability Services or the Centers for Disease Control and Prevention.

\section{REFERENCES}

1. Sleet DA, Moffett DB, Stevens J. CDC's research portfolio in older adult fall prevention: a review of progress, 1985-2005, and future research directions. J Safety Res (2008) 39:259-67. doi:10.1016/j.jsr.2008.05.003

2. Rubenstein LZ. Falls in older people: epidemiology, risk factors and strategies for prevention. Age Ageing (2006) 35:37-41. doi:10.1093/ageing/afl084

3. Tinetti M. Preventing falls in elderly persons. N Engl J Med (2003) 348:42-9. doi:10.1056/NEJMcp020719

4. American Geriatrics Society, British Geriatrics Society, American Academy Of Orthopaedic Surgeons Panel on Falls Prevention. Guideline for the prevention of falls in older persons. J Am Geriatr Soc (2001) 49:664-72. doi:10.1046/j.15325415.2001.49115.x

5. Persad CC, Cook S, Giordani B. Assessing falls in the elderly: should we use simple screening tests or a comprehensive fall risk evaluation? Eur J Phys Rehabil Med (2010) 46:249-59.

6. Stevens JA, Corso PS, Finkelstein EA, Miller TR. The costs of fatal and non-fatal falls among older adults. Inj Prev (2006) 12:290-5. doi:10.1136/ip.2005.011015

7. Roudsari BS, Ebel BE, Corso PS, Molinari NA, Koepsell TD. The acute medical care costs of fall-related injuries among the U.S. older adults. Injury (2005) 36:1316-22. doi:10.1016/j.injury.2005.05.024

8. Ullmann G, Williams HG, Plass CF. Dissemination of an evidence-based program to reduce fear of falling, south Carolina, 2006-2009. Prev Chronic Dis (2012) 9:E103.

9. Kato R, Takagi C, Sakurai N, Hoshi T. Risk factors for falls and survival after falling in elderly people in a community. Nihon Koshu Eisei Zasshi (2012) 59:305-14.

10. Muraki S, Akune T, Ishimoto Y, Nagata K, Yoshida M, Tanaka S, et al. Risk factors for falls in a longitudinal population-based cohort study of Japanese men 
and women: the ROAD study. Bone (2013) 52:516-23. doi:10.1016/j.bone.2012. 10.020

11. Viccaro LJ, Perera S, Studenski SA. Is timed up and go better than gait speed in predicting health, function, and falls in older adults? J Am Geriatr Soc (2011) 59:887-92. doi:10.1111/j.1532-5415.2011.03336.x

12. Landy DC, Mintzer MJ, Silva AK, Dearwater SR, Schulman CI. Hispanic ethnicity and fatal fall risk: do age, gender, and community modify the relationship? J Surg Res (2012) 175:113-7. doi:10.1016/j.jss.2011.02.035

13. Tinetti ME. Prevention of falls and fall injuries in elderly persons: a research agenda. Prev Med (1994) 23:756-62. doi:10.1006/pmed.1994.1130

14. Rizzo J, Baker D, McAvay G, Tinetti M. The cost-effectiveness of a multifactorial targeted prevention program for falls among community elderly persons. Med Care (1996) 34:954-69. doi:10.1097/00005650-199609000-00007

15. Cesari M, Kritchevsky SB, Newman AB, Simonsick EM, Harris TB, Penninx BW, et al. Added value of physical performance measures in predicting adverse health-related events: results from the health, aging and body composition study. J Am Geriatr Soc (2009) 57:251-9. doi:10.1111/j.1532-5415.2008.02126.x

16. Abellan van Kan G, Rolland Y, Andrieu S, Bauer J, Beauchet O, Bonnefoy $\mathrm{M}$, et al. Gait speed at usual pace as a predictor of adverse outcomes in community-dwelling older people an international academy on nutrition and aging (IANA) task force. J Nutr Health Aging (2009) 13:881-9. doi:10.1007/ s12603-009-0246-z

17. Beauchet O, Fantino B, Allali G, Muir SW, Montero-Odasso M, Annweiler C. Timed up and go test and risk of falls in older adults: a systematic review. J Nutr Health Aging (2011) 15:933-8. doi:10.1007/s12603-011-0062-0

18. Studenski S, Perera S, Patel K, Rosano C, Faulkner K, Inzitari M, et al. Gait speed and survival in older adults. JAMA (2011) 305:50-8. doi:10.1001/jama. 2010.1923

19. Huang WW, Perera S, VanSwearingen J, Studenski S. Performance measures predict onset of activity of daily living difficulty in community-dwelling older adults. J Am Geriatr Soc (2010) 58:844-52. doi:10.1111/j.1532-5415.2010. 02820.x

20. Lachman M, Weaver S, Bandura M, Elliott E, Lewkowicz C. Improving memory and control beliefs through cognitive restructuring and self-generated strategies. J Gerontol (1992) 47:293-9. doi:10.1093/geronj/47.5.P293

21. Tennstedt S, Howland J, Lachman M, Peterson E, Kasten L, Jette A. A randomized, controlled trial of a group intervention to reduce fear of falling and associated activity restriction in older adults. J Gerontol B Psychol Sci Soc Sci (1998) 53:384-92. doi:10.1093/geronb/53B.6.P384

22. Healy TC, Peng C, Haynes MS, McMahon EM, Botler JL, Gross L. The feasibility and effectiveness of translating A Matter of Balance into a Volunteer Lay Leader model. J Appl Gerontol (2008) 27:34-51. doi:10.1177/0733464807308620

23. Smith ML, Ahn SN, Sharkey JR, Horel S, Mier N, Ory MG. Successful falls prevention programming for older adults in Texas: rural-urban variations. J Appl Gerontol (2012) 31:3-27. doi:10.1177/0733464810378407

24. Ory MG, Smith ML, Wade AF, Wright JC, Parrish R. Addressing falls in Texas: evidence-based fall prevention programming for older adults. Tex Public Health $J$ (2010) 62:15-20.

25. Cho J, Smith ML, Ahn S, Kim K, Appiah B, Ory MG. Effects of an evidence-based falls risk-reduction program on physical activity and falls efficacy among oldestold adults. Front Public Health (2015) 2:182. doi:10.3389/fpubh.2014.00182

26. Shumway-Cook A, Brauer S, Woollacott M. Predicting the probability for falls in community-dwelling older adults using the timed up \& go test. Phys Ther (2000) 80:896-903.

27. Podsiadlo D, Richardson S. The timed up and go - a test of basic functional mobility for frail elderly persons. J Am Geriatr Soc (1991) 39:142-8.

28. Hutton I, Gamble G, McLean G, Butcher H, Gow P, Dalbeth N. Obstacles to action in arthritis: a community case-control study. Int J Rheum Dis (2009) 12:107-17. doi:10.1111/j.1756-185X.2009.01392.x

29. Benjamins MR, Hummer RA, Eberstein IW, Nam CB. Self-reported health and adult mortality risk: an analysis of cause-specific mortality. Soc Sci Med (2004) 59:1297-306. doi:10.1016/j.socscimed.2003.01.001

30. Idler EL, Angel RJ. Self-rated health and mortality in the NHANES-I epidemiologic follow-up study. Am J Public Health (1990) 80:446-52. doi:10.2105/AJPH. 80.4 .446
31. Appels A, Bosma H, Grabauskas V, Gostautas A, Sturmans F. Self-rated health and mortality in a Lithuanian and a Dutch population. Soc Sci Med (1996) 42:681-9. doi:10.1016/0277-9536(95)00195-6

32. Murata C, Kondo T, Tamakoshi K, Yatsuya H, Toyoshima H. Determinants of self-rated health: could health status explain the association between self-rated health and mortality? Arch Gerontol Geriatr (2006) 43:369-80. doi:10.1016/j. archger.2006.01.002

33. Molarius A, Janson S. Self-rated health, chronic diseases, and symptoms among middle-aged and elderly men and women. J Clin Epidemiol (2002) 55:364-70. doi:10.1016/S0895-4356(01)00491-7

34. Wu S, Wang R, Zhao Y, Ma X, Wu M, Yan X, et al. The relationship between selfrated health and objective health status: a population-based study. BMC Public Health (2013) 13:320. doi:10.1186/1471-2458-13-320

35. Smith ML, Ory MG, Belza B, Altpeter M. Personal and delivery site characteristics associated with intervention dosage in an evidence-based fall risk reduction program for older adults. Transl Behav Med (2012) 2:188-98. doi:10.1007/s13142-012-0133-8

36. Smith ML, Jiang L, Ory MG. Falls efficacy among older adults enrolled in an evidence-based program to reduce fall-related risk sustainability of individual benefits over time. Fam Community Health (2012) 35:256-63. doi:10.1097/FCH. 0b013e318250bdb8

37. Wilcox S, Der Ananian C, Sharpe PA, Robbins J, Brady T. Correlates of physical activity in persons with arthritis: review and recommendations. J Phys Act Health (2005) 2:230-52.

38. Gordon NF, Gulanick M, Costa F, Fletcher G, Franklin BA, Roth EJ, et al. Physical activity and exercise recommendations for stroke survivors: an American heart association scientific statement from the council on clinical cardiology, subcommittee on exercise, cardiac rehabilitation, and prevention; the council on cardiovascular nursing; the council on nutrition, physical activity, and metabolism; and the stroke council. Stroke (2004) 35:1230-40.

39. Center for Disease Control and Prevention, National Center for Injury Prevention and Control, Stopping Elderly Accidents, Dealths, \& Injuries. STEADI Implementation Guide: For Health Care Providers. Washington, DC: Center for Disease Control and Prevention (2012).

Conflict of Interest Statement: The authors declare that the research was conducted in the absence of any commercial or financial relationships that could be construed as a potential conflict of interest.

This paper is included in the Research Topic, "Evidence-Based Programming for Older Adults." This Research Topic received partial funding from multiple government and private organizations/agencies; however, the views, findings, and conclusions in these articles are those of the authors and do not necessarily represent the official position of these organizations/agencies. All papers published in the Research Topic received peer review from members of the Frontiers in Public Health (Public Health Education and Promotion section) panel of Review Editors. Because this Research Topic represents work closely associated with a nationwide evidence-based movement in the US, many of the authors and/or Review Editors may have worked together previously in some fashion. Review Editors were purposively selected based on their expertise with evaluation and/or evidence-based programming for older adults. Review Editors were independent of named authors on any given article published in this volume.

Received: 23 June 2014; accepted: 31 January 2015; published online: 27 April 2015. Citation: Cho J, Smith ML, Shubert TE, Jiang L, Ahn S and Ory MG (2015) Gait speed among older participants enrolled in an evidence-based fall risk reduction program: a subgroup analysis. Front. Public Health 3:26. doi: 10.3389/fpubh.2015.00026

This article was submitted to Public Health Education and Promotion, a section of the journal Frontiers in Public Health.

Copyright (c) 2015 Cho, Smith, Shubert, Jiang, Ahn and Ory. This is an open-access article distributed under the terms of the Creative Commons Attribution License (CC $B Y)$. The use, distribution or reproduction in other forums is permitted, provided the original author(s) or licensor are credited and that the original publication in this journal is cited, in accordance with accepted academic practice. No use, distribution or reproduction is permitted which does not comply with these terms. 